\title{
Strates
}

STRATES Matériaux pour la recherche en sciences sociales

$12 \mid 2006$

Nouvelles tensions impériales et recompositions en Europe centrale, orientale, et CEI

\section{Les eurorégions en Pologne. Un essai d'analyse en termes de district industriel}

\section{Barbara A. DESPINEY-ZOCHOWSKA}

\section{(2) OpenEdition}

\section{Journals}

Édition électronique

URL : http://journals.openedition.org/strates/2322

DOI : $10.4000 /$ strates.2322

ISSN : $1777-5442$

Éditeur

Laboratoire Ladyss

Édition imprimée

Date de publication : 31 décembre 2006

ISSN : 0768-8067

Référence électronique

Barbara A. DESPINEY-ZOCHOWSKA, « Les eurorégions en Pologne. Un essai d'analyse en termes de district industriel », Strates [En ligne], 12 | 2006, mis en ligne le 26 juillet 2007, consulté le 08 septembre 2020. URL : http://journals.openedition.org/strates/2322 ; DOI : https://doi.org/10.4000/ strates. 2322

Ce document a été généré automatiquement le 8 septembre 2020

Tous droits réservés 


\title{
Les eurorégions en Pologne. Un essai d'analyse en termes de district industriel
}

\author{
Barbara A. DESPINEY-ZOCHOWSKA
}

1 Nous nous trouvons aujourd'hui en présence d'un phénomène d'émergence de territoires infranationaux dont beaucoup sont transfrontaliers. Comme l'a écrit Charles-Albert Michalet, «le redécoupage des anciens territoires nationaux ne serait plus un produit de la diplomatie, ni des guerres : il obéirait désormais à une logique d'économie industrielle» (Michalet, 2000). Par ailleurs, la dynamique de l'industrialisation est régionale et «elle n'a pas procédé pays par pays » (Brasseul, 1998). Il convient donc, d'étudier ces territoires infra nationaux dans un cadre européen global et non strictement national, surtout à l'heure de l'élargissement de l'Union européenne (UE) à l'Est. Les systèmes de production régionalisés, regroupés sur un plan spatial, et les réseaux d'entreprises intégrés sur le plan régional peuvent former des noyaux industriels locaux compétitifs. Cet article s'interroge sur la possibilité de renouveau d'un système productif local à la frontière polono-tchécoallemande. D'un point de vue historique, la Silésie est parmi les régions les plus industrialisées d'Europe, après la Belgique, la Ruhr, la Westphalie et l'Alsace. Les résultats de la recherche présentés ici font partie d'un travail commencé au laboratoire Roses de Grenoble, en 1996 , qui se poursuit aujourd'hui dans le cadre du Centre d'économie de la Sorbonne. À l'époque, il s'agissait d'une première étude sur la différenciation à l'Est, faite pour la Datar sur les pôles de croissance, en 1994 et à l'horizon 2015 (Samson et al., 1996). La méthode de calcul adoptée par le Roses a permis d'opérer une différenciation régionale plus fine dans les Peco (Pays d'europe centrale et orientale) et de repérer l'inégale répartition des facteurs de développement durant les cinq premières années de la transition. L'approche de l'espace adoptée relevait, avant tout, de l'économie de la localisation selon laquelle l'espace national est organisé à partir d'une répartition inégale des ressources et des facteurs de production. Quelques éléments relevaient de l'approche de l'économie en tant qu'espace-système, qui intègre la dynamique des espaces, les effets de polarisation et de dominance. Cet article se 
propose de partir d'une troisième approche de l'espace, qui met l'accent sur la notion de district industriel.

De l'industrie lourde aux Systèmes productifs locaux (SPL)

2 Dans le processus de mondialisation, la stratégie de rationalisation de la production des firmes multinationales consiste en une décomposition internationale du processus productif qui induit la perte d'autonomie et la destruction des anciens systèmes productifs nationaux (Andreff, 1996). Mais, cette évolution récente de l'économie mondiale peut apparaître cependant bénéfique pour les économies régionales et locales dans la mesure où elles doivent se resituer dans une perspective globale, au sein de laquelle les entreprises mondiales (firmes multinationales) constituent la principale force créatrice (Amin, Robins, 1992). Le constat est à présent celui d'une compatibilité entre mondialisation et différenciation régionale, voire locale, dans la mesure où les territoires produisent des externalités dont peuvent bénéficier les entreprises dans la perspective d'une adaptation des systèmes locaux à la mondialisation (Pecqueur, 2002).

3 En effet, la mondialisation de l'économie, tout en mettant en relief la nature arbitraire des constructions territoriales et leur relativité historique et culturelle, suggère que de nouveaux aménagements de l'espace sont possibles (Badie, Smouth, 1996). Dans cette situation nouvelle, les traditions et expériences d'un système productif local déterminé devraient pouvoir circuler dans le réseau global, s'incarnant aussi bien dans des produits acceptés sur le marché que dans des idées d'organisation du processus productif, empruntées ou imposées (standardisation, chaîne de production, organisation scientifique du travail ou sous-traitance). Si une expérience locale (un savoir-faire particulier, un matériau spécial, etc.) ne réussit pas à entrer dans le circuit, elle fait alors partie du patrimoine folklorique (Becattini, Rullani, 1995).

4 Les territoires postsocialistes tendent à s'adapter à ces grandes évolutions économiques. Le système productif change avec le changement du système. En effet, le système soviétique privilégiait la centralisation et le gigantisme. Ce sont les grandes unités de production qui furent érigées en symboles identitaires privilégiés de l'ancien régime (Despiney-Zochowska, 1982; Andreff, 1986). Dans ce modèle, l'industrie lourde était le moteur de tout développement et le transfert de technologie, le seul remède au sous-développement. Ce modèle se caractérisait par un faible niveau d'inventions et de découvertes intégrées dans la sphère productive, donc par un faible taux d'innovation (Schneider, 2000). Aujourd'hui, le programme des changements institutionnels qui est au centre des réformes actuelles a pour but la démonopolisation et la décentralisation de l'économie, ainsi que la privatisation quasi totale du secteur d'État. Cette dernière se traduit par une émergence spectaculaire des PMI/PME, moteur de croissance et de la création d'emploi, qui varie toutefois d'un pays à l'autre (Guerraoui, Richet, 2000 ; Duchêne, Rusin, 2002). Ce phénomène de déconcentration à l'Est s'inscrit dans un mouvement économique général, résultant de la contraction de la place des grandes entreprises dans le tissu économique au profit des TPE (Très petites entreprises) et des PME (Petites et moyennes entreprises). La tertiarisation de l'économie constitue l'une des explications de ce phénomène. Depuis 1989, les Peco enregistrent une forte progression de la population des PME, soit par le bas, à travers les dynamiques entrepreneuriales endogènes, soit par le haut, à la suite du fractionnement/ démantèlement des grandes unités de production. Au plan macroéconomique, il s'agit de savoir si les SPL constituent une nouvelle forme d'industrialisation qui favorise l'émergence de petites et moyennes entreprises. 
Paradigme marshallien et modèle polonais du développement

Le district industriel marshallien est fondé sur des économies externes d'agglomération. Il s'agit d'une forme d'organisation industrielle particulière: agglomération géographique de petites entreprises, chacune spécialisées sur une étape $\mathrm{du}$ processus de production, en mettant en place une division externe du travail. Les économies externes décrites par Alfred Marshall sont intra-industrielles, mais celles-ci peuvent également être inter-industrielles et cette distinction recoupe celle qui est faite en économie régionale et urbaine entre les «économies de localisation » et les "économies d'urbanisation", ces deux notions étant réunies sous le vocable «économies d'agglomération " (Marshall, 1920).

Les tenants des premières conceptions du district industriel le présentent comme un complexe productif dont le fonctionnement est soumis au jeu de marché et aux règles de la société civile (Azais, 1997). La notion du système productif local apparaît avec la naissance du terme de production flexible (Piore, Sabel, 1989). L'École française de régulation parle d'un modèle post-fordiste alternatif de « flexibilité offensive » (Boyet, Durand,1993). Cette flexibilité est basée sur la petite dimension des unités de production, la densité des relations entre elles et la rapidité de réponse des entreprises aux nouvelles conditions internes et externes de la zone. La flexibilité, c'est aussi la facilité d'adaptation aux nouvelles technologies. L'avantage concurrentiel des stratégies de spécialisation flexible et des réseaux des petites entreprises repose cependant sur des conditions très spécifiques. Il dépend à la fois d'une demande irrégulière et différenciée et de frais d'établissement obligatoirement limités. Plusieurs facteurs peuvent favoriser un certain degré de reconcentration régionale. Citons, par exemple, la dépendance de certains secteurs des économies d'agglomération et le besoin d'une adaptation plus souple et plus rapide du système productif, du stade de la conception d'un produit à sa fabrication: la proximité géographique peut alors contribuer à la satisfaction de ce besoin. La concentration spatiale peut également être facilitée par la précarité de l'emploi dans de nombreuses zones industrielles traditionnelles, ce qui rend moins nécessaire la recherche d'une main-d'œuvre plus souple et moins coûteuse. L'expérience des districts industriels répond justement à cette nouvelle exigence: il s'agit d'une forme d'industrialisation bien adaptée aux exigences de la flexibilité et qui pourrait aider l'industrialisation des pays émergents et des pays en transition. Dans le cas précis des pays en en transition et pays en voie de développement, on parle à présent de cluster, pour désigner une plus grande diversité de formes que les districts industriels. Cependant, aucun auteur n'a défini rigoureusement les caractéristiques des nouveaux districts industriels de façon à ce que l'on puisse établir aisément des prospectives de leur incidence et de leur croissance dans l'espace et le temps.

7 Le renouveau du concept de district a deux sources : l'une, théorique, basée sur les travaux d'Alfred Marshall, l'autre, empirique, qui est l'émergence, principalement en Italie, d'agglomérations de PME/PMI (Petites et moyennes industries) efficaces, spécialisées dans une étape spécifique d'un processus de production particulier. En effet, c'est l'économiste anglais, Alfred Marshall, qui proposa une lecture différente du marché, qui dépasse le cadre de la concurrence pure et parfaite en partant de l'analyse d'un ensemble d'entreprises regroupées au sein d'un « district industriel ». Il a décrit ainsi la trajectoire sociohistorique spécifique d'un territoire et l'ancrage territorial de l'industrialisation. Le district industriel marshallien ou le Système productif local (SPL), 
formulation plutôt utilisée aujourd'hui, désigne une manière collective de vivre, de penser, de produire, propre à une société, à un espace, à un milieu. Il est une entité socioterritoriale caractérisée par la présence active d'une communauté de personnes et d'une population d'entreprises dans un espace géographique donné. Son trait le plus marquant est son système de valeurs et de pensée relativement homogène, expression d'une certaine éthique du travail et de l'activité, de la famille, de la réciprocité, qui conditionnent en quelque sorte les principaux aspects de la vie (Becattini, 1979). Ce concept fut repris par Antonelli. Pour ce dernier, il ne s'agit pas d'un « idéal-type » de territoire mais plutôt d'une notion "transversale", qui combine des ressources appartenant à des champs disciplinaires différents (Antonelli, 1986). Aujourd'hui, nous assistons à la redécouverte du territoire par ces mêmes économistes. Quant aux géographes, les auteurs californiens proposent une nouvelle interprétation du rôle des économies externes d'agglomération dans la localisation industrielle (Scott, Storper, 1986, 1992). En réfutant le paradigme de coûts de transactions, ils montrent que les économies d'agglomération dérivent de facteurs structurels liés à l'organisation même $\mathrm{du}$ processus de production au sein d'une localité. Ce sont ces économies qui conditionnent le choix de localisation des entreprises. Les auteurs américains et italiens, en associant un siècle plus tard à l'analyse marshallienne de la localisation, une analyse plus fine des modalités de régulation des systèmes industriels contemporains, réintègrent véritablement le rôle des institutions dans l'analyse du développement. Ils ouvrent ainsi la voie à une relecture originale de l'analyse marshallienne.

Émergence des SPL en Pologne

8 Le type de développement qu'on observe aujourd'hui dans les pays de l'Europe centrale et orientale relève justement de l'économie d'agglomération (Brunat, 1995 ; Samson et al., 1996). En Pologne, l'histoire économique du pays et la culture capitaliste inscrite dans la mémoire nationale ont permis l'enracinement d'un esprit commercial et entrepreneurial qui favorise aujourd'hui l'initiative individuelle. Éric Brunat parle même de l'industrialisation diffuse, où la relation essentiellement familiale relève souvent de la relation de type informel (Brunat, 1995). La croissance est étroitement liée en Pologne au dynamisme de petites et moyennes structures émergentes, souvent de nature spontanée. Ces PME produisent une variété de produits en petites séries, et s'adaptent mieux à une demande diversifiée et changeante, au contraire de la production de masse des grandes entreprises fordistes.

Dans la pratique, les districts industriels marshalliens existent à des échelles géographiques variées. Ils peuvent coïncider, quelquefois, avec un quartier particulier dans une ville, ou peuvent s'étendre sur toute une aire métropolitaine (Scott, 1992). Certains travaux semblent confirmer la renaissance des systèmes productifs locaux dans trois métropoles polonaises: Poznan, Lodz et Cracovie (Gruchman et al., 1993, Krätke, 1997). D'autres études montrent que le processus de création et de diffusion des petites et moyennes entreprises est sensible avant tout dans les zones qui jouxtent la frontière allemande, mais il est impossible pour le moment de parler de la formation d'un système productif local. Ce fait ressort également du travail menée à Lodz, où les branches traditionnelles de textile et d'habillement ont vu le plus grand nombre d'entreprises en faillite, mais celles-ci se sont recréées sous forme de petites et moyennes entreprises familiales (Jewtuchowicz, 1998; Duché, 2000), suite à la restructuration des grandes entreprises socialistes qui ont vendu leurs machines et leur équipement à leurs anciens travailleurs. Aujourd'hui, elles représentent seulement $51 \%$ des entreprises recensées dans la ville, « une déspécialisation » et une diversification a 
donc lieu, deux phénomènes pouvant annoncer le renouveau d`un SPL (Garofoli, 1996). L’ancienne tradition industrielle de la ville a permis à la région de se placer en meilleure position par rapport à d'autres régions industrielles polonaises. Cependant, il est difficile, pour le moment, de parler du renouveau de «la culture entrepreunariale ", la pléthore des nouvelles PMI/PME relevant encore trop souvent de ce que l'on appelle "l'économie grise ». Les entreprises, encore nombreuses, sont isolées et exercent leurs activités toutes seules; il n'y a pas de solidarité entre elles. Les entreprises de l'échantillon ne sont pas des entreprises innovantes, car elles sont peu enclines à une coopération avec des organismes de développement et de recherche. Il est, pour le moment, impossible de s'arrêter sur un mode de production précis (Duché, 2000). Selon cet auteur, « l'atmosphère » à la Marshall sera longue à construire. Dans notre étude de cas, nous basons notre hypothèse du renouveau possible d'un SPL dans le secteur du textile-habillement sur le fait que l'expérience de la Troisième Italie concernait au début essentiellement les activités traditionnelles comme le textile, la confection, la maroquinerie et la chaussure placées dans une économie rurale (Martinelli, Schoenberger, 1992). En effet, la majorité des districts italiens se situent près des centres agricoles et une cinquantaine sont spécialisés dans le textile-habillement et la chaussure. Ils représentent $42,5 \%$ de l'emploi industriel et assurent un tiers des exportations italiennes (Vidal, 2001). Pour les auteurs italiens, l'analyse d'A. Marshall permet presque entièrement d'expliquer la dynamique actuelle des régions de la Troisième Italie. Il semble y avoir unanimité pour reconnaître dans le renouveau de l'activité des petites entreprises, une conséquence directe du recours intensif à la soustraitance de la part des entreprises moyennes et grandes (Amin, Robins, 1992). Un récent travail fait par l'OCDE établit la première typologie des districts industriels de Peco (OCDE, 2004).

Coopération transfrontalière en Pologne

10 Un fait important est à retenir : $62 \%$ des habitants des nouveaux membres vivent dans des régions frontalières, alors que ce taux atteint environ $25 \%$ au sein de l'UE à 15 . Pour ne parler que de la Pologne, celle-ci possède à présent des frontières avec les sept États. Cette ouverture internationale confère aux régions polonaises un potentiel de croissance très important. Cet effet ne concerne pas seulement les frontières avec les pays membres de l'Union, mais se fait sentir aussi sur les frontières de l'Est du pays (Borko, 2000 ; Ciechocinska, 2003 ; Despiney, Dressler, 2005). Il en résulte deux formes bien distinctes d'activité, l'une liée au commerce frontalier, l'autre aux eurorégions ${ }^{1}$.

Eurorégions aux frontières de la Pologne

11 Aujourd'hui, on assiste à la généralisation des institutions transfrontalières à travers l'Union, ce qui devrait accélérer une véritable intégration des régions est-européennes à l'Europe. Celle-ci se fait à travers la constitution des eurorégions qui constituaient un test de la volonté d'intégration européenne, déjà avant l'élargissement. Elles donnaient l'opportunité de favoriser à la fois le local et l'arrimage des régions concernées à l'Europe par la création de nouvelles solidarités. Les premiers résultats d'études monographiques, menées dans ces régions transfrontalières, montrent clairement qu'il s'agissait d'une phase initiale du processus de développement d'une coopération durant laquelle les partenaires explorent les caractéristiques de la région frontalière et se familiarisent avec les structures institutionnelles propres aux pays partenaires. Tout un programme de coopération transfrontalière est en cours d'application en Pologne 
depuis quatorze ans par le biais d'eurorégions et de parcs touristiques nationaux. Aujourd'hui, on compte 13 eurorégions situées à ses frontières (carte 1).

L'eurorégion "Neisse » est une des quatre eurorégions situées à la frontière de la Pologne avec l'Allemagne (carte 2) - les trois autres sont «Poméranie», «Pro Europa Viadrina » et "Sprée-Neisse-Bobr ». Située entre les eurorégions "Sprée-Neisse-Bobr » et "Glacensis », elle est considérée comme un laboratoire pour les autres initiatives de ce genre, dans le domaine des statistiques notamment, très peu fiables dans la période de la transition. Ici, une initiative prise par les autorités locales des trois pays limitrophes constitue déjà l'amorce d'une coopération avancée et à long terme. Créée en décembre 1991, elle se compose de 6 Kreise saxons (Bautzen, Kamenz, Löbau-Zittau, Oberlausitz, Görlitz et Hoyerswerda), de 44 communes de la Basse Silésie et de 4 communes appartenant à Lubuskie voïvodie (Pologne), et en ce qui concerne la partie tchèque, des circonscriptions administratives de Liberec, Jablonec, Semily, Ceska Lipa ainsi que la région de Sluknov. L'espace, initialement florissant, regroupant la Basse Silésie, la Bohême du Nord et la Lusace Supérieure, abrite près de 1,5 million d'habitants (725 800 dans la seule partie allemande). Cette frontière, présentée comme ayant un fort potentiel de croissance, dans notre travail fait pour la Datar en 1996 (Samson et al., 1996), connaît une croissance similaire à celle de l'Europe alpine, basée non seulement sur les économies d'agglomération, mais également sur des activités industrielles et tertiaires liées aux activités touristiques. Par ailleurs, il s'agit d'une région historiquement touchée par des revirements multiples dont le dernier acte fut le débat animé sur les enjeux des décrets Benes, à la veille de l'élargissement de l'UE à l'Est (Bazin, 2002). 
Carte 1. Eurorégions localisées aux frontières de la Pologne, Office de statistiques de Voivodie, Wroclaw, 2004.

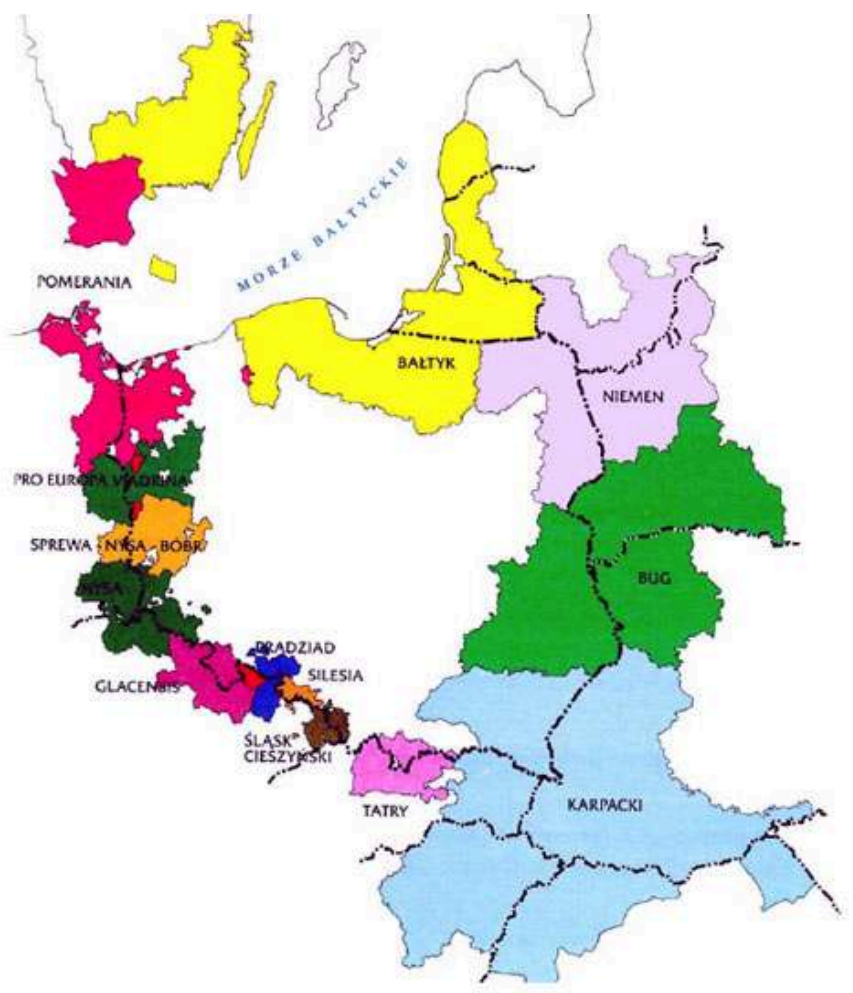

Légende (entre parenthèses la date de la création et éventuellement la traduction polonaise) :

- Eurorégion « Pomeranie » (Pomérania)(décembre 1995)

- Eurorégion «Pro Europa Viadrina » (décembre 1993)

- Eurorégion « Sprée- Neisse - Bobr» (Sprewa-Nysa-Bobr) (septembre 1993)

- Eurorégion « Neisse » (Nysa) (décembre 1991)

- Eurorégion « Glacensis » (décembre 1996)

• Eurorégion « Aïeul » (Adziad)(juillet 1997)

- Eurorégion « Silésie » (Silesia) (Septembre 1998)

- Eurorégion «Silésie de Cieszyn » (mars 1998), (Slask Cieszyrcki)

• Eurorégion « Tatras » (Tatry) (août 1994)

- Eurorégion carpathique (Karpacki) (février 1993)

- Eurorégion «Bug » (septembre 1995)

- Eurorégion « Niemen » (juin 1997)

- Eurorégion «Baltique » (Baltyk) (février 1998). Source : Euro regiony na granicach Polski 2003

(Eurorégions aux frontières de la Pologne en 2003), WUS, Wroclaw, 2004.

Renouveau d'un SPL dans l'eurorégion « Neisse »

Quelles sont aujourd'hui les possibilités du renouveau d'un système productif local qui pourraient engendrer de nouvelles formes de coopération transfrontalière dans l'eurorégion « Neisse » ? C'est une réponse à cette question que se propose de donner ce paragraphe. Plus que jamais, les développements récents dans la région étudiée, semblent en effet confirmer l'hypothèse émise en novembre 1995 (DespineyZochowska, 1997). Un travail de terrain qui reste à faire devrait pouvoir consolider cette assertion, au vu des évolutions récentes ${ }^{2}$.

Une trajectoire régionale

14 Seul le temps long permet d'analyser la dynamique d'un territoire, qui passe par l'observation des hommes dans leurs rapports entre eux et avec les institutions. Dans le 
cas de l'eurorégion « Neisse », nous sommes en présence d'une continuité historique et régionale héritée.

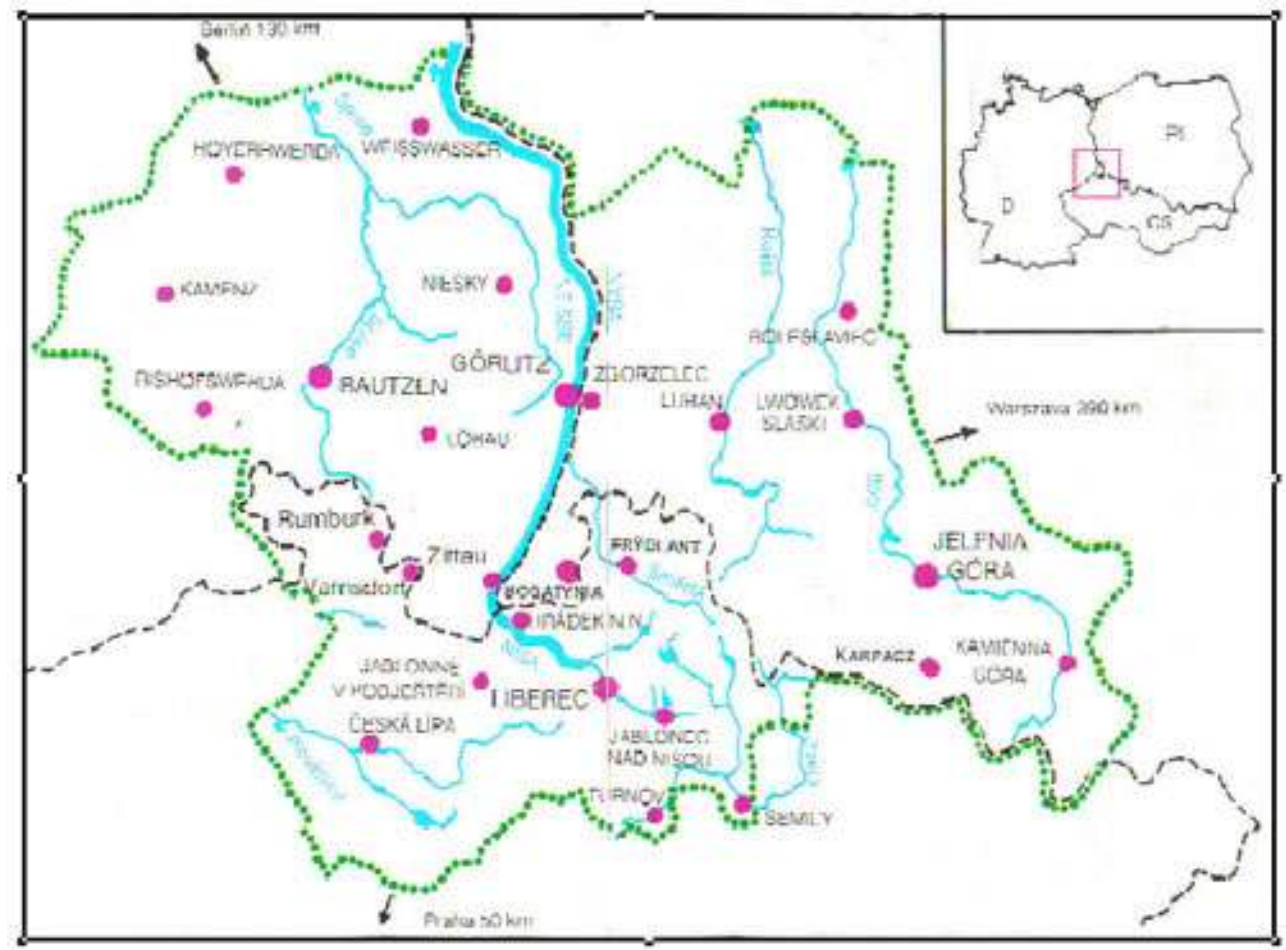

Carte 2. L'eurorégion « Neisse », secrétariat d'Eurorégion, 1996.

En effet, les liens économiques et culturels entre la Saxe, la Silésie polonaise et la Bohême tchèque sont le fruit d'une histoire commune aux trois pays limitrophes dont le lien fondamental est la présence des Allemands au cours des siècles (Jeannin, 1991; Kloczowski et al., 2004). Le développement de l'industrie textile a commencé au $\mathrm{XvI}^{\mathrm{e}}$ siècle à l'initiative des marchands de Haute Allemagne. La Silésie, la Saxe et la Bohême ont vendu massivement à l'Est, jusqu'au milieu du XVII ${ }^{\mathrm{e}}$ siècle, des étoffes de laine de qualité très moyenne. Dès le début du XVII ${ }^{\mathrm{e}}$ siècle, le tissage commence à déborder sur les villages et emploie, au XVIII ${ }^{\mathrm{e}}$ siècle, des familles entières dans la filature. L'impulsion vient de centres qui se distinguent par la richesse de leurs entrepreneurs. Les effets d'entraînement positif de l'industrie rurale sont ici renforcés par le fait que la fabrique de toiles prend place dans une panoplie régionale riche d'industries diverses. Le développement économique fait de la Silésie une région remarquablement avancée en dépit de ses structures féodales (Henderson, 1954). Cette appréciation se fonde surtout sur l'importance internationale des manufactures de toiles qui rivalisent avec les manufactures françaises sur les marchés hispano-américains. La crise structurelle de l'ancienne économie minière $\mathrm{au} \mathrm{XVI}^{\mathrm{e}}$ siècle $\mathrm{y}$ a fait émerger des manufactures de produits colorants, premiers embryons d'une future industrie chimique. La géographie industrielle européenne fut marquée par une dispersion des industries sur les territoires formant des nébuleuses immergées dans le mode rural (Veltz, 1996). La croissance industrielle de la deuxième moitié $d u$ xix et des débuts $d u x^{e}$ siècle se caractérise par une concentration dans des nouvelles régions industrielles du Nord, mais aussi et surtout, par une polarisation autour des grandes agglomérations qui 
deviennent des grands centres de production. C'est bien la polarisation industrielle qui fut le moteur de la concentration spatiale.

La fracture 1945-1989

Le district industriel est régi par un ensemble de valeurs communautaires, religieuses ou par des pratiques corporatistes (Marshall, 1920). Il dispose de connaissances qui lui sont propres, c'est-à-dire non reproductibles et irrémédiablement ancrées sur le territoire (un savoir-faire artisanal spécifique et polyvalent). Il doit créer une " atmosphère industrielle ", facteur d'osmose et de transmission des compétences dans le temps. Avant la seconde guerre mondiale, la Silésie se présentait comme un district industriel au sens marshallien du terme. Après 1945, $43 \%$ des effectifs allemands de l'industrie du chanvre, $49 \%$ des effectifs de la filature, $48 \%$ des effectifs de la toilerie travaillaient dans les régions situées à l'est de l'Oder et de la Neisse, régions allemandes à l'époque (Lepesant, 1997). Le changement des frontières en 1945 et l'expulsion des Allemands ont rompu l'homogénéité du système productif local situé entre la Saxe, la Basse Silésie et la Bohême. L'afflux des réfugiés de l'Est et les migrations de guerre ont modifié les structures de la population de part et d'autre de la ligne Oder-Neisse, sapant les possibilités d'émergence de cultures spécifiques et empêchant le renouveau des traditions régionales anciennes. Par conséquent, durant la période communiste, la frontière Oder-Neisse doit être considérée comme " une coupure " pour reprendre le terme consacré de Claude Courlet, la discontinuité qu'elle provoqua dans la trajectoire $\mathrm{du}$ district industriel ayant pris la forme la plus dure (Courlet, 1988).

Depuis le début de la transition, le rôle de la frontière Oder-Neisse a diamétralement changé. La facilité avec laquelle elle peut être traversée, désormais, permet un contact qui se matérialise dans le développement de courants d'échanges, de migrations, voire de conflits. La frontière devient plus une " couture ", une zone de contact, un lieu de concurrence et de complémentarités. La question reste de savoir si la culture industrielle locale, élément clé de l'édifice institutionnel d'un système productif local, fondée sur les traditions industrielles locales, n'a pas été détruite par cette coupure de 45 ans et dans quelle mesure l'héritage socialiste pèse encore sur la structure des trois espaces régionaux, et sur la réorganisation des relations entre les organisations qui y sont localisées. La transition entraîne une remise en cause radicale des institutions héritées, en même temps qu'une redéfinition des processus économiques et des choix individuels et collectifs (Vercueil, 2000).

Vers une nouvelle identité régionale

De part et d'autre de la frontière Oder-Neisse, le déplacement massif des populations après la seconde guerre mondiale a brouillé l'identification des habitants avec ces régions frontalières durant 45 ans du communisme. Du côté polonais, il s'agissait de terres anciennement allemandes, acquises à la Pologne à la suite de la seconde guerre mondiale. Confisquées, elles furent aussi très vite collectivisées (DespineyZochowska, 1995). L'intégration des travailleurs originaires des terres polonaises de l'Est, posa problème tout au long du système communiste. La part de la population qui a un lieu de résidence identique à son lieu de naissance peut servir de paramètre démographique pour indiquer le degré d'enracinement (Szlachcicowa, 1999). Ce paramètre montre également l'importance des migrations suite à la dernière guerre mondiale, ainsi que celles liées au développement des grands centres industriels, accompagné de la création de grands centres urbains. L'identité régionale ne peut être appréhendée qu'à partir de la mesure des relations entretenues aujourd'hui par les 
populations frontalières avec le lieu de résidence. Deux enquêtes menées dans les régions frontalières allemandes et tchèques nous permettent de se forger une idée sur cette nouvelle identité (Goldhan, 1997 ; Zich, 1997).

En République tchèque, dans les régions proches de la frontière allemande, la part de la population originaire ne représente que 10 à $15 \%$. La majorité de la population a été renouvelée à la suite de l'expulsion des Allemands des Sudètes. Toutes les régions tchèques frontalières montrent (à partir du recensement de 1991) des taux bien plus faibles de la population habitant dans son lieu de naissance, par rapport à la moyenne nationale qui est de $49,9 \%$ (Zich, 1997). Il en est de même pour la population de la partie tchèque de l'eurorégion « Neisse » qui oscille entre $48,9 \%$ et $32,3 \%$ de résidents d'origine. On peut donc supposer que ces populations ont moins de choses en commun avec la localité, la région et son histoire. Peu d'études ont analysé en profondeur les conséquences de ces changements de populations. Les résultats d'une étude sociologique de la population vivant dans les régions à proximité des frontières apportent à ces questions une réponse claire et globalement positive. La structure des réponses montre aussi que les personnes interrogées aiment la région, qu'elles la connaissent bien, sans que pour autant l'intérêt pour les problèmes communs soit suffisamment marquant. Dans toutes les régions proches de l'Allemagne, on observe des différences statistiquement significatives, selon les régions. La différence la plus grande concerne la région qui comporte une agglomération, comme le bassin minier de la Bohême du Nord-Ouest. Les différences les moins marquées sont enregistrées dans les régions intérieures du pays et dans la Forêt de Bohême. Les raisons invoquées par les migrants potentiels sont: l'environnement de mauvaise qualité de la Bohême septentrionale, des raisons familiales, des projets à long terme, l'opportunité de travail ailleurs et le manque de travail. La dégradation de la situation sur le marché du travail depuis 1994 (la date de l'enquête) - principalement dans les districts de Bohême du Nord - a certainement eu un impact négatif sur la stabilité de la population. La situation des petites villes s'est surtout dégradée: s'y manifestent peu à peu les conséquences d'une contraction ou d'une liquidation complète de la production agricole, accompagnée de difficultés de transports. Mais, en dépit des difficultés rencontrées sur le marché du travail, la population tchèque des régions proches de la frontière allemande montre une grande stabilité et a un rapport positif avec son lieu de résidence. Il n'apparaît aucune propension à migrer dont la motivation serait politique ou tiendrait à la nationalité (à l'exception des Tziganes) (Zich, 1997). Pour ce qui est des communes appartenant à l'eurorégion " Neisse ", seules $5 \%$ des personnes interrogées ont déclaré qu'elles voulaient partir dès que possible, $18 \%$ autres n'importe quand et $77 \%$ ne voulaient pas aller ailleurs. Parmi ceux qui veulent partir ou qui pensent partir, $33 \%$ veulent aller dans un autre lieu, mais près de la frontière.

La situation n'est pas identique dans la partie allemande de l'eurorégion. La partie orientale de la Saxe, qui en est partie intégrante, a ressenti l'effondrement de l'économie de l'ex-RDA plus durement que le reste de la Saxe (Goldhan,1997). L'industrie textile a été majoritairement implantée dans les régions déjà très défavorisées : en Haute Lusace, par exemple, l'entreprise de tissage Lautex comptait 16000 employés, Kreise Kamenz 12 entreprises et 1633 salariés, Kreise Oberlausitz 34956 employés. Pour Kamenz seul, la réduction de l'emploi, entre 1989 et 1994, a été de $92 \%$ et pour Oberlausitz de 87,7 \% (Goldhan, 1997). L'effondrement de l'industrie du textile a eu donc un effet catastrophique dans cette partie de l'eurorégion. Contrairement à la situation dans la partie tchèque, on constate une diminution 
sensible de la population avec une tendance au départ des jeunes. Cette hémorragie de la population reflète le déclin d'une région dont l'inégal développement n'est pas seulement imputable à sa situation périphérique mais aux anciens choix de la RDA, qui ont conforté surtout les grands centres urbains comme principaux lieux de la localisation industrielle, obéissant à une logique sectorielle (Grabher, 1994). Plus encore qu'au temps de la RDA, la Saxe orientale se trouve dans une situation périphérique aggravée depuis la réunification allemande. Les études réalisées par l'Institut de géographie de Leipzig montrent qu'à côté du handicap de la Saxe orientale, par rapport au reste de l'Allemagne, cet espace risque de rester le siège de disparités notables. Des travaux plus récents confirment ces affirmations (Lallement, 2004). La dynamique de la population de la ville frontalière de Görlitz montre une tendance au déclin déjà sensible depuis le milieu des années 1950 (de 94 000, elle passe à 64020 en 1996).

21 On peut conclure des données collectées que la partie allemande de l'eurorégion « Neisse» connaît des conditions de localisation défavorables comparativement aux autres territoires de la Saxe. La situation excentrée de l'eurorégion "Neisse » à la frontière orientale de l'État allemand a amené des évolutions particulièrement négatives dans les domaines de l'économie, de l'éducation et de l'emploi. L'absence d'agglomération importante constitue un obstacle au développement. Les transformations structurelles ont amené un taux de chômage élevé un peu partout et particulièrement chez les jeunes et les femmes. Le nombre insuffisant de places de formation par rapport aux demandeurs d'emplois aggrave davantage une situation déjà tendue. La situation critique a provoqué le chômage, le renforcement de l'émigration, surtout des jeunes, avec comme effet le vieillissement de la population résiduelle. On relève que les mesures prises au niveau de l'Allemagne réunifiée pour les infrastructures et les nouveaux investissements se sont concentrées en Saxe, sur Leipzig, Dresde et la partie occidentale du Land et ont rarement touché la Saxe orientale.

Seul, l'espace entre Kamentz (Kamjenc) et Bautzen (Budziszyn), qui fait partie de la Haute Lusace, enregistre un meilleur bilan économique que l'ensemble de la Saxe orientale, ce qui est imputable à la part de la population slave (serbo-lusatienne) et à ses origines chrétiennes. Cette population compte, en effet, environ 100000 personnes dont $20 \%$ sont catholiques et $80 \%$ protestantes. Cette partie de la Saxe qui comprend la minorité serbo-lusatienne semble en effet moins touchée par 50 ans de communisme prussien que la partie ethniquement allemande où les divisions et les solidarités partagées restent complexes à établir, comme le montrent les premiers travaux sur la culture d'entreprise dans une Allemagne unifiée (Müller,1997). Du fait de la restructuration économique, le cadre idéologique rigide s'effondre autant que la solidarité collective, ce qui ne semble pas être le cas pour la minorité évoquée. En effet, les institutionalistes soulignent l'importance des croyances collectives dans le développement des institutions et mettent l'accent sur l'influence de ces mêmes institutions comme entités collectives sur les choix économiques individuels (Denzau, North, 1994). Les individus avec le même passé culturel et les mêmes expériences partagent les mêmes croyances, la même idéologie et les mêmes institutions dans l'interprétation de leur environnement. Les croyances sont des représentations que le système cognitif des individus créent pour interpréter l'environnement; les institutions sont des mécanismes que les individus créent pour structurer l'environnement. Il est démontré, que les groupes ethniques (Juifs, Indiens et Chinois par exemple) qui constituent une minorité dans un État peuvent bénéficier de 
moindres coûts de transaction que le reste de la nation. Un héritage socioculturel commun est un moyen de réduire les divergences dans les représentations collectives, entre les générations en particulier. La possibilité d'apprentissage crée « un effet du sentier " dans les idées et les idéologies, et par la suite, dans des institutions. Les premiers éléments de notre travail sur la frontière polono-tchéco-allemande permettent de confirmer cette thèse. Dans une région allemande en forte dépression comme la Saxe, un seul district semble être en meilleure position au plan du développement : la Haute Lusace. Les meilleurs résultats économiques de celle-ci sont donc à replacer dans une perspective plus large de la dépendance du sentier (path dependency).

Les eurorégions : vers une nouvelle forme de gouvernance?

23 Entre 1945 et 1989, les autorités est-allemandes, polonaises et tchécoslovaques n'ont pas mis en œuvre de politique de développement commune dans les régions frontalières (à l'exception des années 1970). On note donc une faible création d'industries nouvelles et une exploitation intensive du bassin houiller de part et d'autre : en Lusace en RDA et dans la région de Turoszow en Pologne. On constate un fort développement de l'industrie textile, séparément dans chacun des trois pays, ce qui donne aujourd'hui à ces régions frontières, une certaine fragilité (Bafoil, 1995). Sur le plan administratif, le processus de diminution radicale de l'autonomie régionale et locale fut pratiqué de part et d'autre de la frontière. En Allemagne, les Länder furent remplacés par des Kreise émiettés et réduits à n'être que des chambres d'enregistrement des décisions prises à l'échelon central. En Pologne, Edward Gierek procéda à la politique contraire en 1975 en liquidant les powiat (équivalentes des Kreise), échelon administratif intermédiaire, il porta à 49 le nombre des voïvodies.

24 Avec la mondialisation et l'internalisation des activités d'entreprise, un danger se profile cependant pour le renouveau d'un district industriel : la disparition à terme de l'indépendance des unités de production et la transformation en simples soustraitantes de grandes firmes, sûres d'y trouver les compétences techniques nécessaires alliée à une main-d'œuvre bon marché et de pouvoir profiter de leur réseau de clientèle. Dans le cas précis de la sous-traitance, largement utilisée dans le textilehabillement, nous avons affaire à un type de relations interentreprises en forme d'étoile, c'est-à-dire que les relations d'acteurs s'effectuent deux à deux (Courlet, Pecquer, 1991). Il n'y a pas de relations horizontales entre les partenaires d'une même entreprise, et ce type de relation présente souvent pour une PME une relation de dépendance. La présence de mécanismes de régulation est indispensable pour remédier à un tel état de fait (Azaïs, 1997). S'ils ne se mettent pas en place, l'héritage historique considéré comme avantage devient handicap. Dans l'eurorégion "Neisse", l'alignement progressif des salaires est-allemands sur le haut niveau des salaires ouestallemands a fait que les entreprises allemandes du textile-habillement ont largement recouru à la sous-traitance de façonnage des entreprises polonaises et tchèques. Celleci s'est avérée un moyen d'améliorer leur compétitivité à l'échelle internationale (Bensebaa, Brzezinski, 2002). On assiste aujourd'hui, à un recentrage progressif de la production polonaise sur le marché local, au détriment du travail à façon qui se déplace plus à l'Est et une recherche de nouveaux débouchés sur les marchés de l'ex-URSS et les Balkans. Les Polonais et les Tchèques pilotent même ces délocalisations, plus à l'Est, vers la Bélarus, l'Ukraine et la Moldavie, s'imposant comme cotraitants, et non plus comme de simples sous-traitants (IFM, 2004). 

riales publiques sont en voie de décentralisation (Despiney-Zochowska, 2001). On Est-ce que les deux processus, la décentralisation en cours à l'Est et le renouveau
possible des SPL dans cette partie de l'Europe, vont s'autorenforcer ou répondre plutôt
à des dynamiques différentes? En Pologne et en République tchèque, les structures doit donc se demander en quoi et comment l'élément territorial peut contribuer à orienter l'évolution dans le sens d'une meilleure organisation productive. Une chose est sûre : la territorialisation conjointe du système d'entreprise et du système public est susceptible d'assurer, par la coopération de leurs institutions, les conditions de stabilité qui permettront de faire progresser de façon cumulative le savoir-faire jusqu'à développer des capacités locales de création de technologies. Par ailleurs, les districts «à la Marshall» ne peuvent voir le jour que sous l'action des autorités régionales, propriétaires ou simples acteurs, qui développent un réseau d'industries diffuses non spontanées. Dans l'analyse des districts industriels mis en place en Italie, on voit apparaître les premiers éléments d'une gouvernance locale sur ces territoires au travers de l'engagement des autorités publiques locales, du secteur privé et de la société civile (Requier-Desjardins, 2001). Les systèmes productifs locaux peuvent être un outil de politique de développement territorialisé. Avec les SPL, on s'engage vers un nouveau système de concertation entre l'État (l'UE dans le cas des 25) et les collectivités locales. De la décentralisation territoriale dépendent l'autonomie, les pouvoirs et les capacités dont les régions ont besoin pour mener des politiques d'externalités. La décentralisation administrative stimule-t-elle les dynamiques territoriales? Dans notre étude de cas, la réponse est positive. Jusqu'en mars 2001, l'action concertée des trois régions qui composent l'eurorégion « Neisse » était impossible en raison de l'absence de base législative et de stratégie commune pour l'industrie légère dans l'ERN. On manquait aussi d'informations précises sur la situation des entreprises du secteur et sur la diversification possible dans les services. L'existence d'une multitude de petites entreprises mixtes polono-allemandes ne permettait pas de tirer des conclusions sur leur effet d'entraînement dans la restructuration industrielle de la région. Un programme européen d'aide au secteur textile-habillement a été élaboré par les autorités locales à partir de cette date. Il s'agit de la création à Liberec, en mars 2001, d'une eurorégion textile regroupant des associations des producteurs du Brandenbourg, de la Saxe, de la Silésie et de la Bohème : en tout, 1400 entreprises. Cette eurorégion dépasse largement les frontières de la seule eurorégion "Neisse ». D'autres régions transfrontalières polono-allemandes sont concernées comme le Brandenbourg en Allemagne, ainsi que le Lubuskie et la Poméranie occidentale en Pologne. Deux autres eurorégions le sont également: Pro-Europa-Viadrina et NeisseSprée-Bobr. Les trois associations des producteurs locaux du textile-habillement peuvent être considérées comme le prélude à un district (cluster). Un financement européen par Interreg III A à hauteur de 1,5 million d'euros avec des appuis financiers du gouvernement allemand a été est mis en place. Cette nouvelle action stratégique à trois devrait permettre des économies d'échelle et la réduction des coûts de production. Les économies d'échelle liées à la désintégration des marchés de l'Est (Andreff, 1994) pourront ainsi être compensées, la région devant se spécialiser dans les tissus enduits et d'ennoblissements ainsi que dans les tissus de revêtement pour l'automobile. La récente adhésion de la Pologne et de la République tchèque à l'UE 
ouvre certainement de nouvelles possibilités. En effet, selon Paul Krugman, les noyaux régionaux d'exportation dont la cohésion s'explique par les économies marshalliennes externes sont plus propices à se former dans une économie plus intégrée (Krugman, 1993).

\section{BIBLIOGRAPHIE}

Amin A., Robins K., 1992, « Le retour des économies régionales? La géographie mythique de l'accumulation flexible ", in Les régions qui gagnent. Districts et réseaux : les nouveaux paradigmes de la géographie économique, Paris, Puf.

Andreff W., 1986, « The End of Planned Industrialisation Model: What the Lessons for the Third World? », Orstom Conference, Paris, 26-27 of February.

Andreff W., 1994, « De la désintégration à une nouvelle intégration internationale de l'ex-URSS et de l'Europe de l'Est », in M. Lavigne (Ed.), Capitalisme à l'Est. Un accouchement difficile, Paris, Economica.

Andreff W., 1996, «La déterritorialisation des multinationales. Firmes globales et firmes réseau », in Badie B., Smouts M.-C. (Ed.), International sans frontières, Paris, L'Harmattan.

Andreff M., Boudier-Bensebaa F., 2000, « La sous-traitance de façonnage entre l'UE et les pays esteuropéens ", ronéoté, Roses/CNRS.

Antonelli C., 1986, « Technical Districts and Regional Innovation Capacity », Revue d'économie régionale et urbaine, $\mathrm{n}^{\circ} 5$.

Azaïs C., 1997, « Dynamiques territoriales, localisation et systèmes productifs locaux : quelques repères théoriques ", in Palloix Ch., Rizopoulos Y. (Ed.), Firmes et économie industrielle, Paris, L'Harmattan.

Badie B., Smouts M.-C. (Ed.), 1996, International sans frontières, Paris, L'Harmattan.

Bafoil F., 1995, Entre mémoire et attentes : une approche sociologique de la frontière Neisse, Cahiers du ROSES, $\mathrm{n}^{\circ} 1$.

Bazin A., 2002, «Le passé présent : l'enjeu des “décrets Benes” dans la perspective de l'élargissement de l'Union européenne », CERI, 9 décembre.

Becattini G., 1979, « Dal 'settore industriale’ al ‘distretto industriale’ », Rivista di Economia e Politica Industriale, vol. 2.

Becattini G., Rullan E., 1995, « Système local et marché global. Le district industriel » in Rallet A., Torre A. (Ed.)Économie industrielle et économie spatiale, Paris, Economica.

Borko H., 2000, « Paneuropean Economic Co-operation and it's Implications for the Crossborder Economic Co-operation of Poland and Ukraine and Belarus », ronéoté, École centrale de commerce, Varsovie.

Boudier-Bensebaa F., Brezinski H., 2002, « La sous-traitance de façonnage entre l'Allemagne et les pays de l'Est », RECEO, vol. 32, juin. 
Boyer R., Durand J.-P., 1993, L'après-fordisme, Paris, Syros.

Brasseul J., 1998, Histoire des faits économiques, t. 2 : De la révolution industrielle à la Première Guerre Mondiale, Paris, Armand Colin.

Brunat E., 1995, Émergence régionale et dynamique territoriale : essai sur la transformation des économies de type soviétique à partir des exemples russes et polonais, Thèse de doctorat, Université de Grenoble.

Ciechocinska M., 2003, « New Eastern Border as a Process of The EU Enlargement Strategy: A case of Poland (a View from within) », communication au séminaire Recomposition des espaces postcommunistes, Ladyss, Université Paris-X, février.

Courlet C., 1988, « La frontière : coupure ou couture ? ", Économie et Humanisme, n 301, mai-juin.

Courlet C., Pecquer B., 1991, « Systèmes locaux d'entreprises et externalités : un essai de typologie ", RERU, n $3 / 4$.

Denzau A., North D., 1994, « Shared Mental Models: Ideologies and Institutions », Kyklos, vol. 47, Fasc. 1, 3-31.

Despiney-Zochowska B. A., 1982, Les pays socialistes face au nouvel ordre économique international, Thèse, Université de Paris 1 , Paris.

Despiney-Zochowska B. A., 1995, « La restructuration agricole dans les régions frontalières d'Oder-Neisse », Cahiers du ROSES, $n^{\circ} 1$.

Despiney-Zochowska B. A., 1997, «La restructuration industrielle-effets de proximité. Le cas de l'Euro région 'Neisse' », in Struktury przemyslowe w gospodarce, Acta Universitatis Wratislaviensis, $n^{\circ} 1941$.

Despiney B., Dressler W., 2005, « La transition économique et démocratie à la nouvelle frontière de l'UE », communication au 3e colloque PPF « Transition », MSE, 30-31 mai.

Duché G., 2000, « Vers la création d'un nouveau mode de production à Lodz (Pologne). Les freins au développement d'un milieu innovateur », RERU, $\mathrm{n}^{\circ} 1$.

Duchêne G., Rusin P., 2002, « Innovation, mobilité du travail et croissance dans la transition. Le miracle polonais ", in Andreff, W. (Ed.), Analyses économiques de la transition, Paris, La Découverte.

Garofoli G., 1996, « Développement et transformation des systèmes productifs locaux.

Globalisation et coopération interrégionale », Sciences de la société, nº 37, 2, p 83-95.

Gerraoui D., Richet X., 2001, Économies émergentes. Politiques de promotion de la PME. Expériences comparées, Paris, L'Harmattan.

Goldhan G., 1997, « L'euro région “Neisse” définition de la partie allemande de l'euro région », ronéoté, Hochschule für Technik, Wirtschaft und Sozialwesen, Zittau/Görlitz.

Grabher G., 1994, «The Disembedded Regional Economy: The Transformation of East German Industrial Complexes into Western Enclaves », in Amin A., Trift N., p. 177-195.

Gruchman B., et al., 1993, « Milieu innovateur dans une économie en transition », ronéoté, GREMI, Paris.

Henderson W. O., 1954, Britain and Industrial Europe 1750-1870. Studies in British Influence on the Industrial Revolution in Western Europe, Liverpool, University Press.

Institut français de la mode, 2004, Étude sur les implications de la libéralisation des échanges en 2005 dans le secteur textile-habillement, rapport pour la Commission européenne, Paris. 
Jeannin P., 1991, « Dépendance et capacités de développement dans le cadre régional en Europe Centrale à la fin de l'ancien régime ", Cahiers de Varsovie, $\mathrm{n}^{\circ} 22$.

Jewtuchowicz A., Problèmes de développement des petites et moyennes entreprises dans la stratégie de développement régional (exemple de Lodz), ronéoté, Université de Lodz, 1998.

Kloczowski J., Samsonowicz H., Aleksium N., Wandycz P., 2004, Histoire de l'Europe du Centre-Est, Paris, Puf, coll. « Nouvelle Clio ».

Koza A., Podstawowe problemy przemyslu tekstylnego i odziezowego $w E R$ «Nysa », mémoire de maîtrise, Académie économique, Jelenia Gora, 1993.

Krätke S., 1997, Une approche régulationniste des études régionales, L'année de la régulation. Économie, institutions, pouvoirs, Paris, La Découverte, vol. 1.

Krugman P., 1993, « The lessons of Massachusetts for EMU », in Torres F., Giavazzi F., (Ed.), Ajustment and growth in the European Monetary Union, Cambridge, Cambridge University Press.

Lallement R., 2004, « L'économie allemande : transition inachevée et réorientation de la politique économique ", note du Cerfa/Ifri, $\mathrm{n}^{\circ} 17$, novembre.

Lepesant G., 1997, « Development Strategies in cross-border regions of Poland, Germany and Czech Republic », Cerat Report (Université de Grenoble).

Marshall A., 1920, Principles of economics, 8e edition, London, Macmillan.

Martinelli F., Schoenberger E., 1992, « Les oligopoles se portent bien, merci ! », in Benko G., Lipietz A. (Ed.), Les régions qui gagnent. Districts et réseaux : les nouveaux paradigmes de la géographie économique, Paris, Puf.

Michalet Ch.-A., 2000, La séduction des nations ou comment attirer les investisseurs, Paris, Economica.

Müller B., 1997, «Culture, pouvoir et unification allemande dans l'entreprise », in Abélès M., Jeudy H.-P. (Ed.), Anthropologie du politique, Paris, Armand Colin.

OCDE, 2004, Clusters in Transition Economics, Draft, LEED, Paris, 15 July.

Pecqueur B., 2002, «L'organisation spatialisées de la production en France : de nouveaux rapports territoires-entreprises ", ronéoté, séminaire du ministère de la Recherche.

Piore M. J., Sabel C. F., 1989, Les chemins de la prospérité, de la production de masse à la spécialisation souple, Paris, Hachette.

Requier-Desjardins D., 2001, « Décentralisation et dynamiques locales de production dans les pays en développement », Cahiers du GEMDE, $\mathrm{n}^{\circ} 27$.

Samson, et al., 1996, « Pôles de croissance et de décision à l'Est. 1994-2015 », Roses-Grenoble, octobre.

Schneider Ch. M., 2000, « Structure institutionnelle des activités de recherche et transition des économies d'Europe centrale et orientale », Thèse de doctorat, Université Paris-I.

Scott A. J., 1992, «L'économie métropolitaine : organisation industrielle et croissance urbaine », in Benko G., Lipietz A. (Ed.), Les régions qui gagnent, Paris, Puf.

Scott A. J, Storper M. (Ed.), 1986, Production, Work, Territory, Boston, Mass. Allen \& Unvin.

Scott A. J, Storper M., 1992, « Regional Development Reconsidered », in Ernste H., Meier V. (Ed.), Regional Development and Contemporary Industrial Response : Extending Flexible Specialization, Londres, Belhaven. 
Slim A., 1996, « Intégration et désintégration économiques régionales : du CAEM vers de nouvelles unions, Thèse de doctorat, Université Paris-I, septembre.

Szlachcicowa I., 1999, « Tozsamosc mieszkancow polsko-niemieckiego pogranicza w Euro regionie Nysa », Acta Universitatis Wratislaviensis, $\mathrm{n}^{\circ} 2135$.

Veltz P., 1996, Mondialisation, villes et territoires. L'économie d'archipel, Paris, Puf.

Vercueil J., 2000, « La contribution possible d'une "économie institutionnelle du changement" à la compréhension de la transition en Russie », ronéoté, séminaire GTD, Grenoble, 10 mars.

Vidal F., 2001, « Les districts italiens : lieux et milieux porteurs de l'initiative industrielle », communication au Congrès mondial des SPL, Paris, La Villette, 23 et 24 janvier.

Zich F., 1997, Problème d'identité régionale de la population dans les régions tchèques proches des frontières, ronéoté, Liberec, Technicka Univerzita.

\section{NOTES}

1. La coopération transfrontalière contribue à l'intensification des échanges mutuels entre régions de pays différents par l'intermédiaire de deux processus différents : a/ le troc inter-entreprise qui réfère à des contrats à très court terme entre entreprises où la monnaie n'intervient pas ; b/ le commerce transfrontalier, qui est une sorte de commerce spontané et florissant (plutôt illégal) entre les ressortissants originaires des régions concernées (Slim, 1996, Despiney-Zochowska, 1997). Ces deux processus relèvent avant tout de ce que l'on appelle l'« économie grise ». Dès l'ouverture du pays, cette dernière a pris une importance grandissante et a joué un rôle moteur dans le développement des régions concernées. Cependant, l'introduction des visas à la frontière orientale de la Pologne en 1998 a entraîné une baisse du commerce de 50 \% en un an. Cette baisse a concerné également la frontière avec l'Allemagne. C'est la ville de Bialystok, chef-lieu de la Podlasie, qui a le plus souffert de cette décision (plus de $60 \%$ de baisse du commerce frontalier entre 1997 et 1998) alors qu'elle ne fut que de 30 \% à la frontière avec Allemagne (Ciechocinska, 2003 ; Despiney et Dressler, 2005). Le commerce frontalier, qui contribuait à hauteur de 43,7 \% à ce que l'on appelle «l'économie grise », fut en Pologne un moteur du développement local et de la création de nouveaux emplois durant la première phase de la transition. Ce premier gage donné à l'UE à travers la politique de visa sur la frontière orientale a compromis le développement de certaines régions et aggravé le déficit commercial polonais. 2. Ce travail a démarré l'année dernière avec un questionnaire mené auprès d'une dizaine d'entreprises du secteur textile-habillement de la Basse Silésie. Il se fait en coopération avec Case (Varsovie) dans le cadre d'un projet de recherche intitulé « Networking for innovation in a transition country: Potential, opportunities, and policy recommendations ", financé par la Banque mondiale. 


\section{RÉSUMÉS}

Cet article concerne le nouveau phénomène de cluster apparu en Pologne durant la transition, Il prend en compte le rôle des gouvernements locaux. Le principal argument est de comprendre pourquoi la dynamique de développement positif des systèmes productifs locaux basés sur un réseau de firmes de taille petite et moyenne peut frayer le chemin d'un développement durable en Europe centrale et orientale. Une attention particulière est donnée aux aspects régionaux de ces restructurations : notamment à la frontière polono-tchèque allemande. Notre recherche se concentre sur la refondation possible d'un système localisé compétitif dans l'euro-région « Neisse ». Nous nous trouvons confrontés au processus de restructuration de l'industrie textile et de l'industrie du prêt à porter localisée dans cette région depuis le XvII ${ }^{\mathrm{e}}$ siècle. Les systèmes de production régionaux regroupés au plan spatial et intégrant des réseaux de firmes au niveau régional peuvent servir à créer des points locaux de compétition. Les systèmes productifs locaux peuvent être des instruments pour une politique de développement régional. Dans le contexte de l'expansion de l'Europe vers l'Est, ce cas d'étude peut être considéré comme un exemple de mode de gouvernance relativement fructueux.

Barbara Despiney-Zochowska. Euro-regions in poland: attempt of analysis in terms of industrial district This article is dedicated to the new phenomenon of clustering underway in Poland since the transition, taking in consideration the role of local government. The crux of the matter is to understand why the positive development dynamics of local productive systems based on a network of small and medium-sized firms, could be the way for local sustainable development in Central and Eastern Europe. Particular attention is paid to the regional aspect of restructuring: Polish-German-Czech frontier. Our study concentrates on the possible rebirth of a localised competitive system located in "Neisse" Euroregion. We face here the restructuring process of textile and clothing industry located here from xVII Century. Regional production systems grouped together on the spatial level and integrated company networks at the regional level could serve to create local hubs of competition. More, localised productive systems can be an instrument for territorial development policy. In the context of EU expansion eastwards this case study could be considered like success story of new method of governance.

\section{AUTEUR}

BARBARA A. DESPINEY-ZOCHOWSKA

Chercheur, Centre d'économie de la Sorbonne, CNRS/Université de Paris 1, despiney@univ-paris1.fr 